\title{
Feasibility of Protein Biomarkers in the Prediction of Subclinical Doxorubicin Nephrotoxicity in Male Sprague-Dawley Rat
}

\author{
James Eric McDuffie ${ }^{1 *}$, Manisha Sonee ${ }^{2}$, Jing Ma ${ }^{1}$, Frederic Almy ${ }^{2}$, Xuejun Liu ${ }^{3}$, David La ${ }^{1}$ and Sandra Snook ${ }^{1}$
}

${ }^{1}$ Discovery Sciences, Janssen Research \& Development, L.L.C., San Diego, CA, USA

${ }^{2}$ Discovery Sciences, Janssen Research \& Development, L.L.C., Spring House, PA, USA

${ }^{3}$ Immunology Biomarkers, Janssen Research \& Development, L.L.C., San Diego, CA, USA

\begin{abstract}
The feasibility of protein biomarkers in the prediction of subclinical doxorubicin nephrotoxicity was evaluated in male Sprague-Dawley rats during a 2-week study with once-weekly dosing. Doxorubicin $(5,7.5$, and $10 \mathrm{mg} / \mathrm{kg} /$ dose) or $0.9 \%$ Saline was intravenously administered on days 1 and 8 . Urine and serum were collected at various time points. Surviving animals were euthanized on day 14 , and tissues were collected for microscopic examination. Severe clinical signs were observed in the 7.5 and $10 \mathrm{mg} / \mathrm{kg} /$ dose groups. Biomarker data are not reported for these groups because the objective of this study was to evaluate biomarkers at doses not associated with clinical signs. In the $5 \mathrm{mg} / \mathrm{kg}$ group, increased serum concentrations of urea nitrogen were observed on day 14 with concurrent renal histopathology findings which were primarily characterized as slight renal glomerular and tubular injury with mild multi-focal intratubular hyaline casts consistent with protein leakage from damaged glomeruli. Of the various urinary protein biomarkers examined, increased urinary concentrations of albumin was observed on day 7 and increased total protein, albumin and lipocalin-2 were observed on day 14. Taken together, these findings showed that urinary albumin was more sensitive and selective than urinary total protein, lipocalin-2, kidney injury molecule 1 and/or osteopontin in the prediction of progressive doxorubicin-induced glomerular toxicity with secondary renal tubular toxicity in male Sprague-Dawley rats.
\end{abstract}

Keywords: Biomarker; Nephrotoxicity; Glomerulus; Doxorubicin; Rat

Abbreviations: Dox: Doxorubicin; Veh: Vehicle; DIKI: DrugInduced Kidney Injury; GFR: Glomerular Filtration Rate; G: Glomerulus; PT: Proximal Tubule; DT: Distal Tubule; LH: Loop of Henle; sCr: serum Creatinine; BUN: Blood Urea Nitrogen; sChol: serum Cholesterol; sTrig; Triglyceride; uVol; urine Volume; uCr: urinary Creatinine; $\mathrm{uCrCl}$ : urine Creatinine Clearance; uTP: urinary Total Protein; uMAlb: urinary Microalbumin; uAlb: urinary Albumin; uLpn2: urinary Lipocalin-2; uKIM-1: urinary Kidney Injury Molecule 1; uOpn: urinary Osteopontin; ROC: Receiver Operator Characteristic Curve; AUROC: Area Under the ROC Curve; US FDA: United States Food \& Drug Administration; EMA: European Medicines Agency; PMDA, Japan: Pharmaceuticals Medical Devices Agency, Japan

\section{Introduction}

In both preclinical and clinical settings, Drug-Induced Kidney Injury (DIKI) including acute glomerular damage is often undetectable by non-invasive methods such as measurement of Serum Creatinine (sCr) or Blood Urea Nitrogen (BUN) concentrations which represent traditional renal biomarkers [1]. Both $\mathrm{sCr}$ and BUN lack sensitivity and specificity for predicting early alterations in Glomerular Filtration Rate (GFR) which is the best indicator of kidney function.

The United States Food \& Drug Administration (US FDA), European Medicines Agency (EMA) and Pharmaceuticals Medical Devices Agency, Japan (PMDA) have acknowledged the qualification of eight novel urinary protein biomarkers that are highly sensitive and specific for monitoring DIKI progression in rats [1].The qualified renal biomarkers include but are not limited to urinary Total Protein (uTP), urinary Albumin (uAlb) and urinary Kidney Injury Molecule 1 (uKIM-1).The measurement of uTP has been established for monitoring compound-induced acute tubular alterations in preclinical studies in the rat; and uTP outperforms sCr in this context of use. Urinary albumin has been qualified as a biomarker for monitoring compound-induced acute tubular alterations in the rat; and uAlb adds to the interpretation of $\mathrm{sCr}$ and/or BUN concentration changes. Additionally, uAlb often outperforms sCr and/or BUN in the early detection of progressive tubular alterations. Urinary kidney injury molecule 1 is a qualified biomarker for monitoring compound-induced proximal tubular injury in rats; and uKIM-1 may outperform $\mathrm{sCr}$ and/ or BUN.

Phenotypic constitutive osteopontin protein expression in healthy male Sprague-Dawley rat kidneys has been summarized previously [2]. Immunolabeling showed localization of slight renal osteopontin protein staining as early as embryonic day 13; and constitutive osteopontin expression was primarily present in cells in the descending thin limbs of the loop of Henle in the outer medulla and in the papillary surface epithelium in the area of the calyceal fornix. Doxorubicin related induction of osteopontin protein in tubuli with mild dilatation is associated with moderate to severe proteinuria. The potential utility of urinary osteopontin (uOpn) protein concentration measurements for early prediction of doxorubicin nephrotoxicity in male Sprague-Dawley rats has not been previously reported.

The anti neoplastic drug agent, doxorubicin is an experimental

*Corresponding author: James Eric McDuffie, Janssen Research \& Development, L.L.C., 3210 Merryfield Row, San Diego, CA 92121, USA, Tel: 858320 3463; Fax: 858-784-3242; E-mail: jmcduffi@its.jnj.com

Received December 19, 2013; Accepted January 16, 2014; Published January 22, 2014

Citation: McDuffie JE, Sonee M, Ma J, Almy F, Liu X, et al. (2014) Feasibility of Protein Biomarkers in the Prediction of Subclinical Doxorubicin Nephrotoxicity in Male Sprague-Dawley Rat. J Mol Biomark Diagn 5: 165. doi:10.4172/21559929.1000165

Copyright: () 2014 McDuffie JE, et al. This is an open-access article distributed under the terms of the Creative Commons Attribution License, which permits unrestricted use, distribution, and reproduction in any medium, provided the original author and source are credited 
nephrotoxicant which has been shown previously to induce experimental glomerular toxicity with secondary renal tubular toxicity in Sprague-Dawley [2] and Han Wistar [3] rats. The rat model of doxorubicin nephrotoxicity is the most commonly used to evaluate mechanisms involved in compound-induced progressive glomerular injury associated with significant proteinuria [4-6]. Stemming from the qualification of novel DIKI biomarkers in rats, it is paramount to further understand the value of multiplex measurement of urinary protein concentrations of uTP, uAlb, uKIM- 1 and uOpn for monitoring early, acute glomerular injury with secondary renal tubular injury in rats. In this study, various traditional and novel protein biomarkers were evaluated to determine their sensitivity and specificity in the prediction of progressive, subclinical doxorubicin nephrotoxicity in male Sprague-Dawley rats.

\section{Materials and Methods}

\section{Statement of ethical approval}

All animal procedures were conducted in an Association for Assessment and Accreditation of Laboratory Animal Care International-accredited facility under an Institutional Animal Care and Use Committee-approved protocol. Standard procedures and conditions for animal care, feeding, and maintenance of room, caging, and environment were used.

\section{Experimental design}

Male Sprague-Dawley rats, 7- to 8-weeks-old and weighing 229 to 277 grams were purchased from Charles River Laboratories (Sacramento, CA). Animals were randomly assigned to either a control (Vehicle, Veh: $0.9 \%$ Saline) group or test article (nephrotoxicant: doxorubicin) group(s) as shown in Table 1.

Doxorubicin hydrochloride (Dox, Sigma-Aldrich, St. Louis, MO) was formulated in $0.9 \%$ Sodium ChlorideInjecti on, USP (Baxter, Deerfield, IL). On study days 1 and 8 , rats were administered via a lateral tail vein a bolus intravenous injection of either doxorubicin $(5$, 7.5 , or $10 \mathrm{mg} / \mathrm{kg} /$ dose) or $0.9 \%$ Saline. Study termination was on day 14. Water was provided ad libitum via water bottles. On days 6 and 13, animals were fasted (overnight) and housed individually in metabolism cages for approximately 18 hours. During the time of fasting, urine samples were collected on wet ice and the total urine volumes collected per animal were recorded. On day 7 and at the time of necropsy, blood was collected via tail vein and the posterior vena cava, respectively in serum separator tubes. Blood was processed to obtain serum. Urine and serum aliquots were stored at approximately $-80^{\circ} \mathrm{C}$ until time of analysis. On day 14, animals were humanely euthanized by $\mathrm{CO}_{2}$ inhalation followed by exsanguination. Heart, kidney, and liver were collected for histopathological examination.

\section{Serum and urinary biomarker analysis}

Siemens Advia 1800 automated chemistry system and reagents

\begin{tabular}{|c|c|}
\hline Experimental group & Animal \# \\
\hline $\begin{array}{c}\text { Control: } \\
\text { Vehicle }(0.9 \% \text { Saline })\end{array}$ & $1-5$ \\
\hline Nephrotoxicant test article: & \\
\hline Doxorubicin $(5 \mathrm{mg} / \mathrm{kg} /$ dose $)$ & $21-25$ \\
\hline Doxorubicin $(7.5 \mathrm{mg} / \mathrm{kg} /$ dose $)$ & $31-35$ \\
\hline Doxorubicin $(10 \mathrm{mg} / \mathrm{kg} /$ dose $)$ & $41-45$ \\
\hline
\end{tabular}

On study days 1 and 8 , male Sprague-Dawley rats were administered via a lateral tail vein a bolus intravenous injection of either Vehicle $(0.9 \%$ Saline) or Doxorubicin. Study termination was on day 14.

Table 1: Experimental group assignments.
(Siemens Corporation, Washington, DC) were used to measure serum concentrations of creatinine ( $\mathrm{sCr}$ ), urea nitrogen (BUN), cholesterol (sChol), and triglyceride (sTrig) and urinary concentrations of creatinine (uCr), total protein (uTP) and microalbumin (uMAlb). Urine creatinine clearance $(\mathrm{uCrCl})$ was calculated using the equation: urine volume $(m L) \times$ urinary creatinine concentration $(\mathrm{mg} / \mathrm{dL}) /$ serum creatinine concentration $\mathrm{mg} / \mathrm{dL} \times 1080$ minutes divided by body weight $(\mathrm{Kg})$. Sector Imager type instrument and Kidney Injury Panel 1 (rat) kit reagents (Meso Scale Discovery, Gaithersburg, MD) were used to simultaneously measure urinary concentrations of kidney injury molecule 1 (uKIM-1), lipocalin-2 (uLpn2), osteopontin (uOpn), and albumin (uAlb) in urine samples that were diluted (at 1:10) in Diluent 29 solution provided in the Kidney Injury Panel 1 (rat) kit. Urinary biomarker concentrations were normalized to concurrent urinary creatinine concentrations.

\section{Histopathology}

Representative heart, liver and kidney (left and right) samples were fixed in $10 \%$ neutral buffered formalin for approximately 48 hours, processed, embedded in paraffin, sectioned at $4-\mu \mathrm{m}$, mounted on glass slides, de-parraffinized, and stained with hematoxylin and eosin for subsequent microscopic evaluation. Severity scores were assigned based on the classification of microscopic evidence of renal injury; and the distribution and increased numbers of renal cells affected were assigned a quantitative severity score: $0=$ no abnormality noted, $1=$ slight (minimal, $<25 \%), 2=$ mild $(25-50 \%), 3=$ moderate $(>50 \%)$ and $4=$ severe $(>75 \%)$.

\section{Statistical analysis}

Group data are presented as mean \pm SE except where noted. Where applicable, fold changes from control are indicated. As appropriate, comparison between two groups at the same time point was made by unpaired Student's ttest to reveal statistical significance (indicated as ${ }^{* * *} \mathrm{p}<0.001,{ }^{* *} \mathrm{p}<0.01$ and $\left.{ }^{*} \mathrm{p}<0.05\right)$ using GraphPad Prism software version 6 for Windows (GraphPad Software, San Diego, CA).

\section{Biomarker performance analysis}

Receiver-Operator Characteristic Curve (ROC) analysis was employed to compare the biomarker performance, whereby the area under the ROC curve (AUROC) was used to determine the accuracy of BUN, sCr, uCr, uTP/uCr, uMAlb/uCr, uAlb/uCr, uLpn2/uCr,uKIM-1/ $\mathrm{uCr}$ and $\mathrm{uOpn} / \mathrm{uCr}$ in the prediction of progressive treatment doxorubicin related nephrotoxicity. Performance depended on how well the biomarker separated the experimental groups tested into those with and without changes in biomarker concentrations on day 7 and the sum of individual animal renal injury severity scores on day 14 (Table 7). AUROC represented biomarker performance according to the following scale: $\geq 0.90$ (high), $0.80-0.90$ (moderate), $0.70-0.80$ (mild) and $\leq 0.60$ (poor). The statistical significance of the AUROC values were assessed by $\mathrm{P}$ values, which represent the probability of rejecting the null hypothesis that the AUROC curve is 0.5 , indicating that there is no predictive power at all.

\section{Results}

\section{Clinical observations and mortality}

Numerous clinical signs including oral discharge, ventral staining, and decreased activity were observed in the 7.5 and $10 \mathrm{mg} / \mathrm{kg} / \mathrm{dose}$ doxorubicin group animals. Due to the severity of clinical signs, 1 of 5 animals in the $7.5 \mathrm{mg} / \mathrm{kg} /$ dose group and 5 of 5 animals in the $10 \mathrm{mg} / \mathrm{kg} /$ dose group were humanely euthanized prior to the scheduled necropsy 
Citation: McDuffie JE, Sonee M, Ma J, Almy F, Liu X, et al. (2014) Feasibility of Protein Biomarkers in the Prediction of Subclinical Doxorubicin Nephrotoxicity in Male Sprague-Dawley Rat. J Mol Biomark Diagn 5: 165. doi:10.4172/2155-9929.1000165

on study day 14 . The $7.5 \mathrm{mg} / \mathrm{kg}$-treated animal was euthanized on day 7 while the $10 \mathrm{mg} / \mathrm{kg} /$ dose-treated animals were euthanized on days 7 , 9, and 10. Biomarker and histopathology data are not reported for the 7.5 and $10 \mathrm{mg} / \mathrm{kg} /$ dose groups because the objective of this study was to evaluate the feasibility of serum and urinary protein biomarkers in the prediction of progressive nephrotoxicity at doses not associated with remarkable clinical signs.

\section{Traditional biomarker changes}

On day 7 , increased concentrations of sChol (1.3-fold, $\mathrm{p}=0.037)$ and decreased $\mathrm{uVol}(0.6$-fold, $\mathrm{p}=0.045)$ were measured, while on day 14 , increased concentrations of $\mathrm{uTP} / \mathrm{uCr}$ (74.3-fold, $\mathrm{p}=0.0006)$, sTrig (5.4-fold, $\mathrm{p}=0.008$ ), sChol (4.6-fold, $\mathrm{p}=0.002$ ), $\mathrm{BUN}$ (2.1-fold, $\mathrm{p}=0.002$ ), $\mathrm{uCr}$ (1.7-fold, $\mathrm{p}=0.02)$ and decreased $\mathrm{uVol}(0.4$-fold, $\mathrm{p}=0.026)$ were measured in the $5 \mathrm{mg} / \mathrm{kg} / \mathrm{dose}$ group relative to the concurrent control group (Figures 1 and 2; Tables 2 and 3).

\section{Novel urinary biomarker changes}

In the $5 \mathrm{mg} / \mathrm{kg} / \mathrm{dose}$ group, increased concentrations of uMAlb/ $\mathrm{uCr}$ (432.6-fold, $\mathrm{p}=0.043$ on day 7 and 62.5 -fold, $\mathrm{p}=0.047$ on day 14 ) were detected using an automated chemistry analyzer (Figure 3 and Table 4). Changes for uAlb, uLpn2, uKIM-1 and uOpn were detected simultaneously using a 4-plex assay and are summarized in Table 5. For statistical analysis for uAlb, the ULOD $(50,600 \mathrm{ng} / \mathrm{mL})$ was used when values were above the ULOD. On days 7 and 14 , the uAlb concentrations were respectively increased ( $>8.6$-fold, $\mathrm{p}=0.0005$ and $>33.1$-fold, $\mathrm{p}=0.036$ ). Concentrations of $\mathrm{uKIM}-1 / \mathrm{uCr}$ were decreased ( 0.5 -fold, $\mathrm{p}=0.0004)$ on day 7 and increased on day 14 , but the difference was not statistically significant $(5.3$-fold, $\mathrm{p}=0.12)$. Concentrations of $\mathrm{uLpn} 2 / \mathrm{uCr}$ were not statistically increased on day 7 (1.3-fold, $\mathrm{p}=0.14)$, but were significantly increased on day 14 ( 4.1 -fold, $\mathrm{p}=0.02$ ).

\section{Renal histopathology findings}

Once weekly intravenously administered doxorubicin $(5,7.5$ or 10 $\mathrm{mg} / \mathrm{kg} /$ dose) induced dose-dependent renal glomerular injury (Table 6). There were no doxorubicin related histopathology findings in the liver or heart at the doses tested in this study.

\section{Discussion}

In this study, the feasibility of protein biomarkers in the prediction of subclinical doxorubicin nephrotoxicity was evaluated in male Sprague-Dawley rats [2-6]. Of the doses tested, only the doxorubicin ( $5 \mathrm{mg} / \mathrm{kg} /$ dose) and concurrent control (vehicle) group animals were evaluated for detection of novel renal biomarker concentration changes because higher doses of doxorubicin $(7.5$ and $10 \mathrm{mg} / \mathrm{kg} / \mathrm{dose})$ were associated with clinical signs of systemic toxicity and histopathology findings. Changes in the 7.5 and $10 \mathrm{mg} / \mathrm{kg} / \mathrm{dose}$ groups included hyaline casts suggesting protein leakage from damaged glomeruli;
A

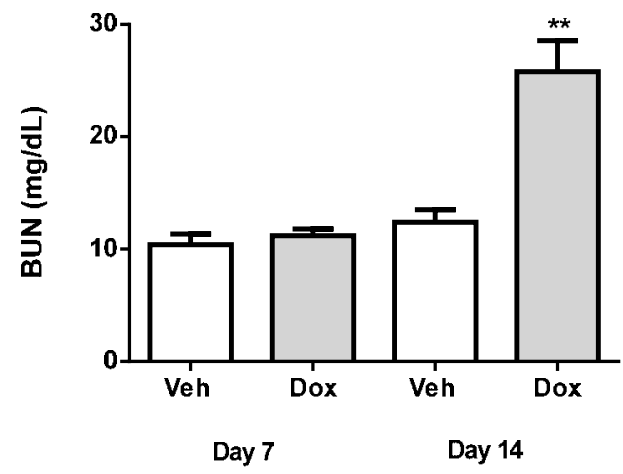

C

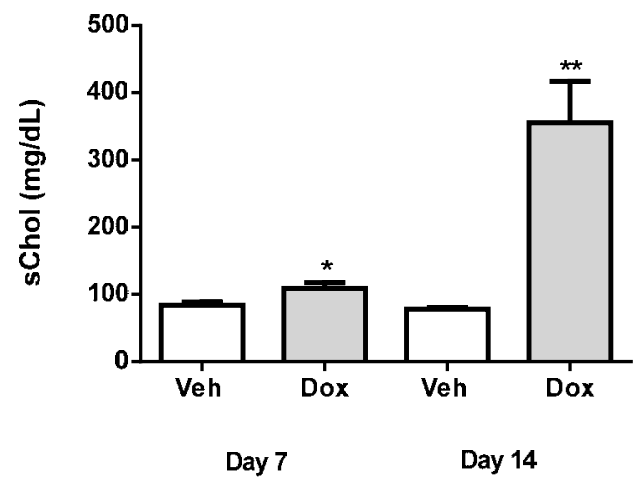

B

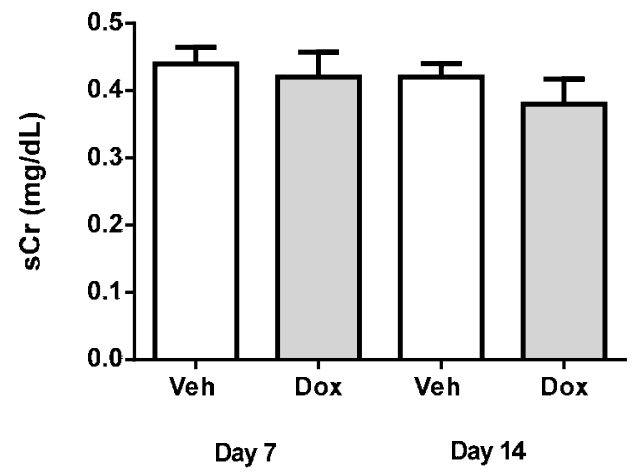

D

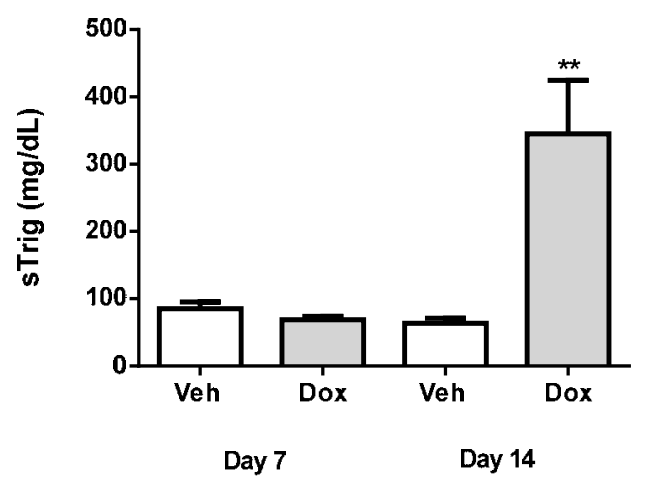

Figure 1: Serum biomarker (blood urea nitrogen, BUN; serum creatinine, sCr; serum cholesterol, sChol; serum triglycerides, sTrig) concentration changes in male Sprague-Dawley rats administered Vehicle (Veh, $0.9 \%$ saline) or Doxorubicin (Dox, $5 \mathrm{mg} / \mathrm{kg} / \mathrm{dose}$ ) and samples collected on study days 7 and 14 . Values significantly different from vehicle control are indicated as ${ }^{* *} p<0.01$ or ${ }^{*} p<0.05$. 
Citation: McDuffie JE, Sonee M, Ma J, Almy F, Liu X, et al. (2014) Feasibility of Protein Biomarkers in the Prediction of Subclinical Doxorubicin Nephrotoxicity in Male Sprague-Dawley Rat. J Mol Biomark Diagn 5: 165. doi:10.4172/2155-9929.1000165

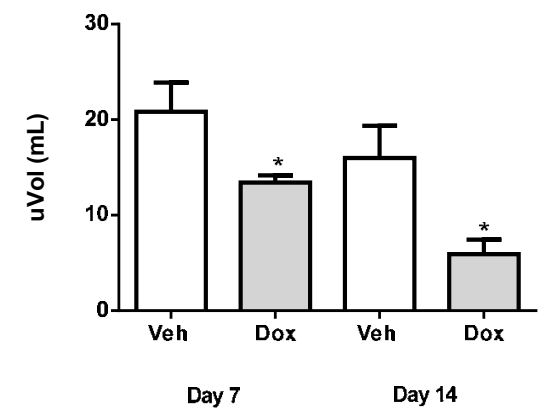

C

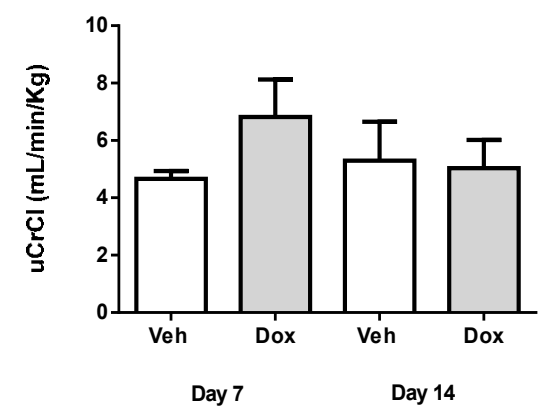

B

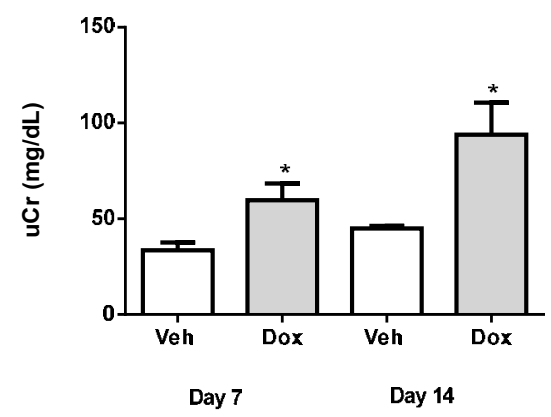

D

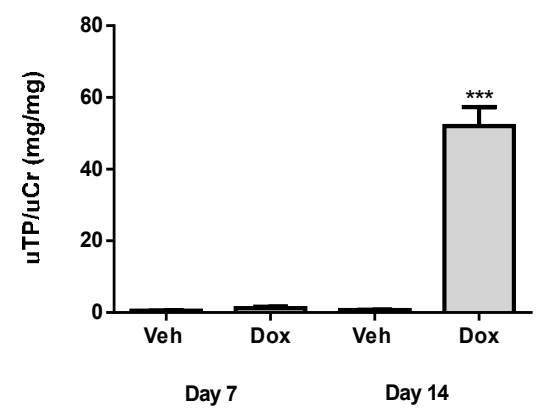

Figure 2: Urinary parameter (urine volume, uVol; urinary creatinine, uCr; urine creatinine clearance, uCrCl; urinary total protein, uTP [normalized to concurrent urinary creatinine concentrations, $\mathrm{uCr}$ ) changes in male Sprague-Dawley rats administered Vehicle (Veh, 0.9\% saline) or Doxorubicin (Dox, $5 \mathrm{mg} / \mathrm{kg} / \mathrm{dose}$ ) and samples collected on study days 7 and 14 . Values significantly different from vehiclecontrol are indicated as ${ }^{* * *} p<0.001$ or ${ }^{*} p<0.05$.

\begin{tabular}{|c|c|c|c|c|c|}
\hline & \multicolumn{5}{|c|}{ Study Day 7} \\
\hline & Animal \# & BUN (mg/dL) & $\mathrm{sCr}(\mathrm{mg} / \mathrm{dL})$ & sChol (mg/dL) & sTrig (mg/dL) \\
\hline \multirow{6}{*}{ 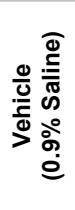 } & 1 & 7 & 0.4 & 98 & 88 \\
\hline & 2 & 10 & 0.4 & 74 & 104 \\
\hline & 3 & 12 & 0.5 & 89 & 76 \\
\hline & 4 & 12 & 0.4 & 74 & 52 \\
\hline & 5 & 11 & 0.5 & 84 & 106 \\
\hline & $\bar{X} \pm$ SE & $10.4 \pm 0.9$ & $0.4 \pm 0.0$ & $83.8 \pm 4.6$ & $85.2 \pm 10.0$ \\
\hline \multirow{7}{*}{ 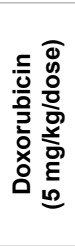 } & 21 & 9 & 0.4 & 119 & 70 \\
\hline & 22 & 12 & 0.4 & 101 & 87 \\
\hline & 23 & 11 & 0.3 & 78 & 59 \\
\hline & 24 & 12 & 0.5 & 129 & 58 \\
\hline & 25 & 12 & 0.5 & 117 & 70 \\
\hline & $\bar{X} \pm$ SE & $11.2 \pm 0.6$ & $0.4 \pm 0.0$ & $108.8 \pm 8.9$ * & $68.8 \pm 5.2$ \\
\hline & Fold $\Delta$ & 1.1 & 1.0 & 1.3 & 0.8 \\
\hline \multirow{7}{*}{ 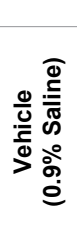 } & \multicolumn{5}{|c|}{ Study Day 14} \\
\hline & 1 & 9 & 0.4 & 84 & 49 \\
\hline & 2 & 12 & 0.4 & 70 & 83 \\
\hline & 3 & 12 & 0.4 & 74 & 68 \\
\hline & 4 & 16 & 0.5 & 84 & 44 \\
\hline & 5 & 13 & 0.4 & 78 & 74 \\
\hline & $\bar{X} \pm$ SE & $12.4 \pm 1.1$ & $0.4 \pm 0.0$ & $78.0 \pm 2.8$ & $63.6 \pm 7.4$ \\
\hline \multirow{7}{*}{ 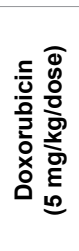 } & 21 & 26 & 0.5 & 363 & 247 \\
\hline & 22 & 28 & 0.3 & 341 & 365 \\
\hline & 23 & 16 & 0.3 & 141 & 94 \\
\hline & 24 & 26 & 0.4 & 506 & 504 \\
\hline & 25 & 33 & 0.4 & 428 & 515 \\
\hline & $\bar{X} \pm$ SE & $25.8 \pm 2.8^{\star \star}$ & $0.4 \pm 0.0$ & $355.8 \pm 60.9^{* *}$ & $345.0 \pm 79.8^{* *}$ \\
\hline & Fold $\Delta$ & 2.1 & 1.0 & 4.6 & 5.4 \\
\hline
\end{tabular}

Table 2: Serum biomarker concentration changes on days 7 and 14. 
Citation: McDuffie JE, Sonee M, Ma J, Almy F, Liu X, et al. (2014) Feasibility of Protein Biomarkers in the Prediction of Subclinical Doxorubicin Nephrotoxicity in Male Sprague-Dawley Rat. J Mol Biomark Diagn 5: 165. doi:10.4172/2155-9929.1000165

Page 5 of 9

\begin{tabular}{|c|c|c|c|c|c|}
\hline & \multicolumn{5}{|c|}{ Study Day 7} \\
\hline & Animal \# & uVol (mL) & $\mathrm{uCr}(\mathrm{mg} / \mathrm{dL})$ & $\begin{array}{c}\mathrm{uCrCl} \\
(\mathrm{mL} / \mathrm{min} / \mathrm{Kg})\end{array}$ & $\begin{array}{l}\text { uTP/uCr } \\
\text { (mg/mg) }\end{array}$ \\
\hline \multirow{6}{*}{ 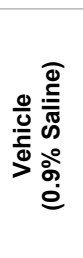 } & 1 & 21.20 & 29.7 & 5.2 & 0.3 \\
\hline & 2 & 32.40 & 20.7 & 5.5 & 0.6 \\
\hline & 3 & 15.30 & 44.7 & 4.1 & 0.6 \\
\hline & 4 & 17.12 & 31.9 & 4.1 & 0.5 \\
\hline & 5 & 18.19 & 40.5 & 4.4 & 0.5 \\
\hline & $\bar{X} \pm \mathbf{S E}$ & $20.8 \pm 3.0$ & $33.5 \pm 4.2$ & $4.7 \pm 0.3$ & $0.5 \pm 0.1$ \\
\hline \multirow{7}{*}{ 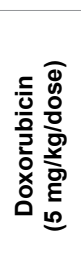 } & 21 & 12.46 & 50.1 & 5.7 & 0.7 \\
\hline & 22 & 12.48 & 31.3 & 3.4 & 0.8 \\
\hline & 23 & 14.14 & 68.2 & 11.4 & 0.4 \\
\hline & 24 & 11.95 & 82.6 & 6.5 & 1.4 \\
\hline & 25 & 16.02 & 66.4 & 7.0 & 2.8 \\
\hline & $\bar{X} \pm \mathrm{SE}$ & $13.4 \pm 0.7^{*}$ & $59.7 \pm 8.8^{*}$ & $6.8 \pm 1.3$ & $1.2 \pm 0.4$ \\
\hline & Fold $\Delta$ & 0.6 & 1.7 & 1.4 & 3.4 \\
\hline & \multicolumn{5}{|c|}{ Study Day 14} \\
\hline \multirow{6}{*}{ 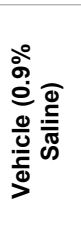 } & 1 & 17.37 & 47.8 & 6.3 & 0.8 \\
\hline & 2 & 28.38 & 46.5 & 10.2 & 0.9 \\
\hline & 3 & 11.38 & 43.0 & 3.5 & 0.6 \\
\hline & 4 & 9.34 & 47.1 & 2.7 & 0.7 \\
\hline & 5 & 13.45 & 40.0 & 3.8 & 0.5 \\
\hline & $\bar{X} \pm \mathbf{S E}$ & $16.0 \pm 3.4$ & $44.9 \pm 1.5$ & $5.3 \pm 1.4$ & $0.7 \pm 0.1$ \\
\hline \multirow{7}{*}{ 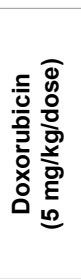 } & 21 & 2.51 & 145.5 & 6.4 & 55.9 \\
\hline & 22 & 11.47 & 51.5 & 3.9 & 55.8 \\
\hline & 23 & 5.19 & 102.0 & 12.7 & 31.0 \\
\hline & 24 & 4.16 & 107.3 & 6.7 & 59.5 \\
\hline & 25 & 6.22 & 63.2 & 7.8 & 57.8 \\
\hline & $\bar{X} \pm \mathbf{S E}$ & $5.9 \pm 1.5^{*}$ & $93.9 \pm 16.8^{*}$ & $5.0 \pm 1.0$ & $52.0 \pm 5.3^{* \star \star}$ \\
\hline & Fold $\Delta$ & 0.4 & 2.1 & 0.9 & \begin{tabular}{|l|}
74.3 \\
\end{tabular} \\
\hline
\end{tabular}

Values significantly different from Vehicle $(0.9 \%$ Saline) control are indicated as $^{* * *} p<0.001$ or ${ }^{*} p<0.05$. Urinary total protein (uTP) was normalized to concurrent urinary creatinine concentrations $(\mathrm{uCr})$.

Table 3: Urinary parameter changes on days 7 and 14.

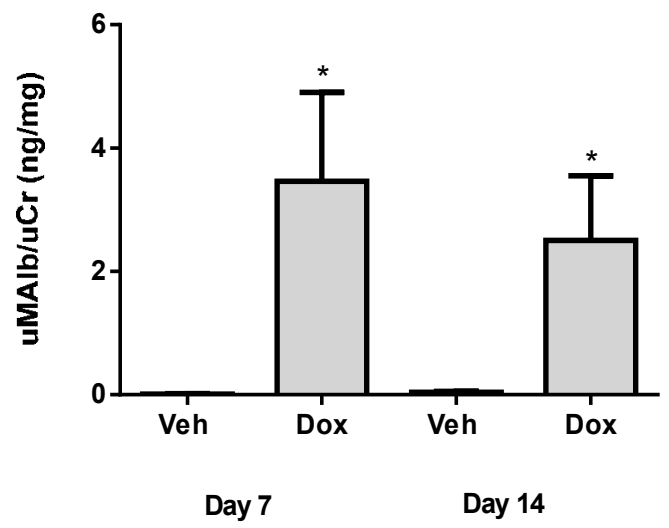

Figure 3: Changes in urinary microalbumin (uMAlb) [normalized to concurrent urinary creatinine $(\mathrm{uCr})$ concentrations] in male Sprague-Dawley rats administered Vehicle (Veh, $0.9 \%$ saline) or Doxorubicin (Dox, $5 \mathrm{mg} / \mathrm{kg} / \mathrm{dose}$ ) and samples collected on study days 7 and 14 .Values significantly different from vehicle control are indicated as ${ }^{*} p<0.05$.

electron microscopy would be needed to better characterize the renal lesions [3,5].

As summarized in Table 7, to evaluate the predictive power of renal biomarkers observed on Day 7 for diagnosing glomerular injury, we calculated the AUROCs and associated $P$ values for $\mathrm{BUN}$ and $\mathrm{sCr}$ concentrations and normalized concentrations of uTP, uMAlb, uAlb, uLpn2, uKIM-1 and uOpn, and compared values with and the sum of the individual animal histopathology severity scores. On day 7, BUN (Mean AUROC $=0.62$, AUC $\mathrm{p}=0.65$ ) and $\mathrm{sCr}$ (Mean AUROC: 0.44 AUC $\mathrm{p}=0.82$ ) were unchanged in the doxorubicin group relative to the

\begin{tabular}{|c|c|c|c|}
\hline & & Study Day 7 & Study Day 14 \\
\hline & Animal\# & \multicolumn{2}{|c|}{ uMAlb/uCr (ng/mg) } \\
\hline \multirow{6}{*}{ 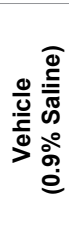 } & 1 & 0 & 0 \\
\hline & 2 & 0 & 0 \\
\hline & 3 & 0.04 & 0.07 \\
\hline & 4 & 0 & 0.06 \\
\hline & 5 & 0 & 0.05 \\
\hline & $\bar{X} \pm \mathbf{S E}$ & $0.008 \pm 0.008$ & $0.04 \pm 0.01$ \\
\hline \multirow{7}{*}{ 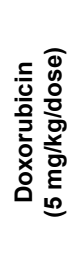 } & 21 & 1.2 & 0.8 \\
\hline & 22 & 3.19 & 6.4 \\
\hline & 23 & 1.47 & 1.5 \\
\hline & 24 & 2.42 & 0.8 \\
\hline & 25 & 9.04 & 3.0 \\
\hline & $\bar{X} \pm \mathrm{SE}$ & $3.46 \pm 1.4^{*}$ & $2.5 \pm 1.1^{*}$ \\
\hline & Fold $\Delta$ & 432.5 & 62.5 \\
\hline
\end{tabular}

Values significantly different from Vehicle $(0.9 \%$ Saline) control are indicated as ${ }^{*} p<0.05$. Urinary microalbumin (uMAlb) concentrations were normalized to concurrent urinary creatinine $(\mathrm{uCr})$ concentrations.

Table 4: Urinary microalbumin changes on days 7 and 14.

\begin{tabular}{|c|c|c|c|c|c|}
\hline & \multicolumn{5}{|c|}{ Study Day 7} \\
\hline & $\begin{array}{c}\text { Animal } \\
\#\end{array}$ & $\begin{array}{l}\text { uAlb/uCr } \\
\text { (ng/mg) }\end{array}$ & $\begin{array}{c}\text { uLpn2/uCr } \\
\text { (ng/mg) }\end{array}$ & $\begin{array}{l}\text { uKIM-1/uCr } \\
\text { (ng/mg) }\end{array}$ & $\begin{array}{c}\text { uOpn/uCr } \\
\text { (ng/mg) }\end{array}$ \\
\hline \multirow{6}{*}{ 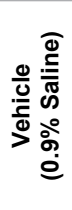 } & 1 & 12743.8 & 170.6 & 0.9 & 3.9 \\
\hline & 2 & 10130.9 & 236.2 & 1.3 & 9.4 \\
\hline & 3 & 15509.5 & 318.4 & 0.8 & 5.3 \\
\hline & 4 & 9748.2 & 181.7 & 1.1 & 5.0 \\
\hline & 5 & 8619.3 & 193.2 & 1.0 & 7.2 \\
\hline & $\bar{X} \pm \mathrm{SE}$ & $11350.0 \pm 1240$ & $220.0 \pm 27.0$ & $1.0 \pm 0.1$ & $6.2 \pm 1.0$ \\
\hline \multirow{7}{*}{ 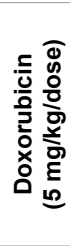 } & 21 & $>105857.7 \S$ & 370.3 & 0.5 & 5.0 \\
\hline & 22 & $>108817.2 \S$ & 280.8 & 0.5 & 5.1 \\
\hline & 23 & 38808.0 & 246.6 & 0.5 & 5.7 \\
\hline & 24 & $>107431.0 \S$ & 231.7 & 0.6 & 4.8 \\
\hline & 25 & $>126500.0 \S$ & 269.5 & 0.4 & 7.0 \\
\hline & $\bar{X} \mathbf{\pm} \mathbf{S E}$ & $>97483.0 \pm 15137 \S^{* * *}$ & $280.0 \pm 24.2$ & $0.5 \pm 0.0^{* * *}$ & $5.5 \pm 0.4$ \\
\hline & Fold $\Delta$ & $>8.6 \S$ & 1.3 & 0.5 & 0.9 \\
\hline & \multicolumn{5}{|c|}{ Study Day 14} \\
\hline \multirow{6}{*}{ 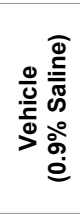 } & 1 & 8078.3 & 175.8 & 0.6 & 2.7 \\
\hline & 2 & 8993.5 & 325.1 & 1.0 & 7.9 \\
\hline & 3 & 13690.8 & 216.2 & 0.7 & 2.6 \\
\hline & 4 & 11170.9 & 142.8 & 1.0 & 2.0 \\
\hline & 5 & 11382.1 & 148.9 & 0.8 & 3.8 \\
\hline & $\bar{X} \mathbf{\pm}$ SE & $10663.0 \pm 985.2$ & $201.8 \pm 33.4$ & $0.8 \pm 0.1$ & $3.8 \pm 1.1$ \\
\hline \multirow{7}{*}{ 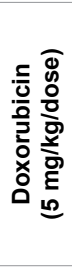 } & 21 & $>34776.6 \S$ & 775.9 & 3.3 & 4.7 \\
\hline & 22 & $>98252.4 \S$ & 678.1 & 2.5 & 4.2 \\
\hline & 23 & $>49607.8 \S$ & 378.6 & 0.5 & 3.2 \\
\hline & 24 & $>47157.5 \S$ & 936.3 & 3.1 & 3.9 \\
\hline & 25 & $>80063.3 \S$ & 1334.1 & 11.89 & 5.2 \\
\hline & $\bar{X} \mathbf{\pm} \mathbf{S E}$ & $\begin{array}{c}>353567.0 \pm \\
136273.0 \S^{*}\end{array}$ & $\begin{array}{l}820.6 \pm \\
157.3^{* *}\end{array}$ & $4.2 \pm 1.9$ & $4.2 \pm 0.4$ \\
\hline & Fold $\Delta$ & $>33.1 \S$ & 4.1 & 5.3 & 1.1 \\
\hline
\end{tabular}

Urinary biomarkers (urinary albumin, uAlb; urinary lipocalin-2, uLpn2; urinary kidney injury molecule 1, KIM-1; urinary osteopontin, uOpn) were normalized to concurrent urinary creatinine $(\mathrm{uCr})$ concentrations. §Doxorubicin-induced increased uAlb concentrations were above the upper limit of assay detection. Values significantly different from Vehicle $(0.9 \%$ Saline $)$ control are indicated as ${ }^{* * *} p<0.001,{ }^{* *} p<0.01$ or ${ }^{*} p<0.05$.

Table 5: Changes in novel urinary biomarker on days 7 and 14 . 
Citation: McDuffie JE, Sonee M, Ma J, Almy F, Liu X, et al. (2014) Feasibility of Protein Biomarkers in the Prediction of Subclinical Doxorubicin Nephrotoxicity in Male Sprague-Dawley Rat. J Mol Biomark Diagn 5: 165. doi:10.4172/2155-9929.1000165

Page 6 of 9

\begin{tabular}{|c|c|c|c|c|c|}
\hline \multirow{3}{*}{ Microscopic histopathology finding } & \multicolumn{5}{|c|}{ Experimental group/ Animal \# } \\
\hline & \multicolumn{5}{|c|}{ Vehicle (0.9\% Saline) } \\
\hline & 1 & 2 & 3 & 4 & 5 \\
\hline \multirow[t]{3}{*}{ Multi-focal tubular dilatation and basophilia (cortex) } & 0 & 0 & 0 & 0 & 1 \\
\hline & \multicolumn{5}{|c|}{ Doxorubicin (5 mg/kg/dose) } \\
\hline & 21 & 22 & 23 & 24 & 25 \\
\hline Multi-focal decrease of Bowman's space and mesangial expansion (glomeruli) & 1 & 1 & 1 & 1 & 1 \\
\hline Multi-focal hyaline casts (intratubular) & 2 & 2 & 1 & 2 & 2 \\
\hline Multi-focal tubular vacuolization with fine hyaline droplets (cortico-medullary junction) & 1 & 1 & 1 & 1 & 1 \\
\hline Focal tubular degeneration with individual cell necrosis (cortico-medullary junction) & 1 & 1 & 1 & 1 & 1 \\
\hline Multi-focal tubular dilatation and basophilia (cortex) & 0 & 1 & 0 & 1 & 0 \\
\hline
\end{tabular}

Doxorubicin related renal histopathology severity scores were based on the classification of microscopic evidence of renal injury observed at termination on study day 14 ; and the distribution and increased numbers of renal cells affected were assigned a quantitative severity score: $0=$ no abnormality noted, $1=$ slight (minimal, $<25 \%$ ), $2=$ mild $(25-50 \%), 3=$ moderate $(>50 \%)$ and $4=$ severe $(>75 \%)$. On study days 1 and 8 , male Sprague-Dawley rats were administered via a lateral tail vein a bolus intravenous injection of either doxorubicin or saline.

Table 6: Incidence and severity of microscopic evidence of doxorubicin related renal injury.

\begin{tabular}{|c|c|c|c|c|c|c|c|c|c|c|}
\hline & & \multicolumn{2}{|c|}{ Serum biomarker } & \multirow[b]{2}{*}{$\mathrm{Cr}^{*}(\mathrm{mg} / \mathrm{dL})$} & \multirow[b]{2}{*}{$\operatorname{TP}^{s^{*}}(\mathrm{mg} / \mathrm{mg})$} & \multirow[b]{2}{*}{ MAlb $^{\S^{*}}(\mathrm{ng} / \mathrm{mg})$} & \multirow[b]{2}{*}{$A l b^{s^{*}}(\mathrm{mg} / \mathrm{mg})$} & \multirow[b]{2}{*}{$\operatorname{Lpn}^{\S}(\mathrm{mg} / \mathrm{mg})$} & \multirow[b]{2}{*}{ KIM-1 $1^{*}(\mathrm{mg} / \mathrm{mg})$} & \multirow[b]{2}{*}{$O p n^{\S}(\mathrm{mg} / \mathrm{mg})$} \\
\hline & & BUN (mg/dL) & $\mathrm{Cr}(\mathrm{mg} / \mathrm{dL})$ & & & & & & & \\
\hline \multicolumn{2}{|c|}{ Nephron specificity } & \multirow[b]{2}{*}{$\mathrm{N}-\mathrm{S}$} & \multirow[b]{2}{*}{$\mathrm{N}-\mathrm{S}$} & \multirow[b]{2}{*}{$\mathrm{N}-\mathrm{S}$} & \multirow[b]{2}{*}{$\mathrm{G}, \mathrm{PT}$} & \multirow[b]{2}{*}{ G, PT } & \multirow[b]{2}{*}{ G, PT } & \multirow{2}{*}{$\begin{array}{c}\text { G, PT } \\
\text { DT }\end{array}$} & \multirow[b]{2}{*}{ PT } & \multirow[b]{2}{*}{ G, PT, DT, LH } \\
\hline Animal \# & $\begin{array}{c}\text { Severity } \\
\text { Score }^{\Sigma}\end{array}$ & & & & & & & & & \\
\hline 1 & 0 & 7 & 0.4 & 29.7 & 0.3 & 0.00 & 12744 & 170.6 & 0.9 & 3.9 \\
\hline 2 & 0 & 10 & 0.4 & 20.7 & 0.6 & 0.00 & 10131 & 236.2 & 1.3 & 9.4 \\
\hline 3 & 0 & 12 & 0.5 & 44.7 & 0.6 & 0.04 & 15510 & 318.4 & 0.8 & 5.3 \\
\hline 4 & 0 & 12 & 0.4 & 31.9 & 0.5 & 0.00 & 9748 & 181.7 & 1.1 & 5.0 \\
\hline 5 & 1 & 11 & 0.5 & 40.5 & 0.5 & 0.00 & 8619 & 193.2 & 1.0 & 7.2 \\
\hline 21 & 5 & 9 & 0.4 & 50.1 & 0.7 & 1.20 & 105858 & 370.3 & 0.5 & 5.0 \\
\hline 22 & 6 & 12 & 0.4 & 31.3 & 0.8 & 3.19 & 108817 & 280.8 & 0.5 & 5.1 \\
\hline 23 & 4 & 11 & 0.3 & 68.2 & 0.4 & 1.47 & 38808 & 246.6 & 0.5 & 5.7 \\
\hline 24 & 6 & 12 & 0.5 & 82.6 & 1.4 & 2.42 & 107431 & 231.7 & 0.6 & 4.8 \\
\hline 25 & 5 & 12 & 0.5 & 66.4 & 2.8 & 9.04 & 126500 & 269.5 & 0.4 & 7.0 \\
\hline \multicolumn{2}{|c|}{ AUROC } & 0.62 & 0.44 & 0.88 & 0.84 & 1.00 & 1.00 & 0.80 & 1.00 & 0.42 \\
\hline \multicolumn{2}{|c|}{ AUC $P$ value } & 0.65 & 0.83 & 0.09 & 0.15 & 0.009 & 0.009 & 0.22 & 0.009 & 0.77 \\
\hline
\end{tabular}

On study days 1 and 8, male Sprague-Dawley rats were administered via a lateral tail vein a bolus intravenous injection of either doxorubicin or saline. Study termination was on day 14 . Urinary protein biomarker concentrations were normalized to concurrent urinary creatinine (uCr) concentrations on day $7 .{ }^{*}$ Doxorubicin related increased urinary protein biomarker concentrations exhibited predictive power for early, non-invasive prediction of progressive glomerular toxicity with secondary renal tubular reabsorption impairment. §Doxorubicin-induced increased urinary albumin (uAlb) concentrations were above the upper limit of assay detection. ${ }^{\Sigma}$ Values represent the sum of the individual animal histopathology severity scores. AUROC represented biomarker performance according to the following scale: $\geq 0.90$ (high), $0.80-0.90$ (moderate), $0.70-0.80$ (mild) and $\leq 0.60$ (poor). The $P$ value represents the probability of rejecting the null hypothesis that the AUROC curve is 0.5 , indicating that there is no predictive power at all. Other abbreviations: blood urea nitrogen BUN; total protein, TP; microalbumin, MAlb; lipocalin-2, Lpn2; kidney injury molecule 1, KIM-1; osteopontin, Opn.

Table 7: Biomarker performance in the prediction of subclinical doxorubicin related nephrotoxicity.

concurrent control group; therefore, neither BUN nor sCr predicted doxorubicin related progressive nephrotoxicity which was evident on day 14 as verified using light microscopy. Doxorubicin related azotemia was recognized by increased concentrations of BUN (2.1fold, $\mathrm{p}=0.002$ ) in the absence of increased $\mathrm{sCr}$ on day 14 (Figure $1 \mathrm{~A}$ and Table 2).

Of the various urinary biomarkers evaluated per the day 7 samples, increased urinary albumin (uMAlb: AUC $\mathrm{p}=0.009$ and uAlb: $\mathrm{p}=0.009$, Figure 3 and Table 4 ) exhibited high predictive power (Mean AUROC=1.0) for prediction of progressive doxorubicin nephrotoxicity characterized as primary glomerular injury and secondary tubular injury (Table 6). Increased concentrations of uMAlb and uAlb/ uCron day 7 were $>432.5$-fold and $>8.6$-fold elevated, respectively and were more sensitive than either sCr or BUN. Elevated uAlb/uCr concentrations were indicative of high permeability for albumin in the renal glomerulus; and uAlb is a qualified biomarker which outperforms $\mathrm{sCr}$ and/or BUN for monitoring compound-induced acute tubular alterations in rats [1,7]. The present findings demonstrate clearly that uAlb may be increased with glomerular injury and subsequent leakage or tubular injury and decreased reabsorption. Thus, uAlb cannot be used feasibly as a fit-for-purpose biomarker in the absence of other biomarkers to localize the nephron specific site of injury. However, increased uMAlb and uAlb concentrations in the absence of increased uKIM-1 concentrations would be indicative of a primary glomerular lesion. Likewise, the increased $\mathrm{uTP} / \mathrm{uCr}(3.4$-fold, $\mathrm{p}=0.13)$ detected on day 7 was not statistically significant because the minimal glomerular injury and minimal tubular epithelium injury seen in this study likely were below the threshold needed to induce acute changes in $\mathrm{uTP} / \mathrm{uCr}$. However, ROC analysis showed that $\mathrm{uTP} / \mathrm{uCr}$ (Mean AUROC $=0.84$, AUC $\mathrm{p}=0.15$ ) indeed moderately out performed $\mathrm{sCr}$ and $\mathrm{BUN}$ in the prediction of progressive glomerular toxicity with secondary renal tubular reabsorption impairment.

Doxorubicin related renal injury was associated with elevated uMAlb/uCr (62.5-fold, $\mathrm{p}=0.05)$, uTP/uCr (74.3-fold, $\mathrm{p}=0.05)$ and $\mathrm{uAlb} / \mathrm{uCr}(>33.1$-fold, $\mathrm{p}=0.05)$ on day 14 . These findings are consistent with previous studies that demonstrated the utility of uTP and uAlb concentrations for monitoring glomerular injury with renal tubule reabsorption impairment in rats and humans [1-7]. Both uTP and uAlb have been qualified as rat urinary renal biomarkers for the prediction of compound-induced renal dysfunction, tissue injury response and tissue 
leakage [1,7]. Investigation with lower doxorubicin doses may lend additional insights relative to the sensitivity for urinary concentrations of total protein and/or albumin in the early detection of doxorubicin related nephrotoxicity.

Previous studies showed that plasma triglyceride concentrations are higher in nephrotic than in analbuminemic rats following single intravenous doxorubicin $(4 \mathrm{mg} / \mathrm{kg})$ administration despite a similar increase in hepatic triglyceride secretion [8]. On day 7, significant related sTrig concentration changes were not observed following administration of doxorubicin at either 5 (Figure 1D and Table 2), 7.5 or $10 \mathrm{mg} / \mathrm{kg} /$ dose (data not shown). Concentrations of sTrig were elevated (5.4-fold, $\mathrm{p}=0.058$ ) by day 14 following once-weekly administration of doxorubicin $(5 \mathrm{mg} / \mathrm{kg} /$ dose $)$. On day 7 , concentrations of sChol were increased at 1.3 -fold $(\mathrm{p}=0.037)$ and by day 14 were elevated 4.6 -fold, $\mathrm{p}=0.002$ ). These sTrig and sChol changes observed on day 7 occurred in the absence of histologic evidence of liver injury on day 14 . Blood triglyceride concentrations are higher in the male Sprague-Dawley rat doxorubicin nephrotoxicity model than in male Sprague-Dawley rats with congenital analbuminemia [8]. Our findings are consistent with the theory of increased cholesterol and triglyceride concentrations in blood and oliguria which are compensatory responses to significant proteinuria which results in decreased urine volume (also observed in this study on both days 7 and 14) and decreased colloid osmotic pressure. The present study demonstrated the utility of serum lipidemia as hallmark serum chemistry changes indicative of doxorubicin nephrotoxicity in the rat.

Other renal tissue injury response urinary biomarkers that have been qualified for monitoring compound-induced renal injury in the rat include UKIM-1, urinary clusterin, urinary renal papillary antigen 1 and urinary trefoil factor 3 [1,7]. In male Sprague-Dawley rats, KIM1 is a transmembrane protein that is strongly upregulated primarily but not exclusively in proximal tubule epithelial cells upon injury and subsequently shed into the urine $[1,7,9]$. Measurably increased uKIM-1 protein has specificity and sensitivity for use as a qualified biomarker to monitor compound-induced proximal tubular injury in rats. Based on the similarities for nephrotoxicant-induced KIM-1 protein expression characteristics observed in the rat, uKIM-1 is also considered qualified as a clinical bridging biomarker to monitor renal safety in clinical studies on a "case-by-case" basis following the identification of tubular injury in rats $[1,7]$. In the present rat study, the concentrations of $\mathrm{uKIM}-1 / \mathrm{uCr}$ were decreased ( 0.5 -fold, $\mathrm{p}=0.0004$ ) on day 7 (Figure $4 \mathrm{C}$ and Table 5); and the minimal and focal degree of tubular degeneration with individual cell necrosis observed on day 14 (Table 6) was likely below the threshold needed to induce statistically significant elevations in $\mathrm{uKIM}-1 / \mathrm{uCr}$ (Table 5). Decreased concentrations of uKIM-1/uCr on day 7 appeared predictive (Mean AUROC=1.0, AUC $\mathrm{p}=0.009$ ) for progressive doxorubicin-induced kidney injury (Table 7). Concentrations of uKIM- $1 / \mathrm{uCr}$ were increased on day 14 , but the difference was not statistically significant $(5.3-$ fold, $\mathrm{p}=0.12)$ relative to controls. These findings indicate that the mechanisms responsible for doxorubicin related tubular toxicity (Table 6) and renal dysfunction may be characterized by proteinuria (Figure $2 \mathrm{D}$ and Table 3 ). Taken together, the doxorubicin related increases in $\mathrm{uAlb} / \mathrm{uCr}$ without concomitant and significantly increased uKIM-1/uCr appeared to be diagnostic of glomerular injury in the absence of notable microscopic tubular injury in male Sprague-Dawley rats.

The expression, roles, receptors, and regulation of osteopontin in kidney have been summarized previously [2]. Osteopontin is synthesized mostly in bone and epithelial tissues; its expression in normal kidneys depends on the species, age, and gender. In rats, renal osteopontin is expressed as early as embryonic day 13 and is primarily present in cells in the descending thin limbs of the loop of Henle in the outer medulla and in the papillary surface epithelium in the area of the calyceal fornix. Induction of osteopontin may occur in glomeruli. Proteinuria often appears upstream to increased nephron-specific osteopontin expression and concurrent acute nephrotoxicity particularly in the thick ascending limb of the loop of Henle, distal tubules, and collecting ducts. During chronic tubulointerstitial injury, co-localization of ED-1 (CD68, macrophage marker), vimentin (intermediate filament marker), proliferating cell nuclear antigen (PCNA, cellular proliferation marker) and increased osteopontin in renal tubuli could potentially serve as a biomarker signature indicative of the cellular regenerative response to protein-induced tubular injury [10]. To date, uOpn has not been qualified as a biomarker to monitoring drug-induced kidney injury in rat preclinical studies or in the clinical setting. In this study, following repeat dose administration of doxorubicin $(5 \mathrm{mg} / \mathrm{kg} /$ dose $)$ or vehicle ( $0.9 \%$ Saline), the uOpn concentrations were similar on both days 7 and 14. Similar to uKIM-1/ $\mathrm{uCr}$, the doxorubicin related minimal tubular epithelium injury seen in this study likely was below the threshold needed to induce relevant changes in $\mathrm{uOpn} / \mathrm{uCr}$.

Lipocalin-2 which is also known as Neutrophil GelatinaseAssociated Lipocalin (NGAL) is a $25-\mathrm{kDa}$ protein that was initially identified bound to gelatinase in neutrophil granules and functions principally to regulate bacterial growth [11]. The function and localization of the Lpn2/NGAL/24p3 receptor (24p3R) in rat renal epithelium have been described previously. It is constitutively expressed in various tissues and induced in epithelial cells. In male Wistar rats, $24 \mathrm{p} 3 \mathrm{R}$ is expressed in apical plasma membranes of the distal nephron and mediates high-affinity protein endocytosis in renal cells and is associated with protein endocytosis and nephrotoxicity. Urinary lipocalin-2 is filtered by the glomeruli and reabsorbed in the proximal tubules. Proteinuria appears generally upstream to increased uOpn. To date, uLpn 2 has not been qualified as a biomarker for monitoring druginduced kidney injury in rat preclinical studies or in the clinical setting. In this study, following repeat dose administration of doxorubicin (5 $\mathrm{mg} / \mathrm{kg} / \mathrm{dose}$ ), uLpn $2 / \mathrm{uCr}$ was increased (4.1-fold) on day 14 ; however, this increase may be a secondary response to the doxorubicin related marked proteinuria. Furthermore, the hyaline casts noted in the doxorubicin-treated rat renal tubules are further evidence of protein endocytosis upregulated in these animals relative to the group control animals. Additional chronic studies compound would be warranted to further evaluate progressive doxorubicin-induced marked proteinuria and concomitant renal damage.

Previous reports showed that Tamm-Horsfall protein (also known as uromodulin) is constitutively expressed in Sprague-Dawley rat distal convoluted tubules [12]. In the present study, doxorubicin related renal histopathology findings were characterized by the presence of multifocal hyaline casts in intratubular lumens was consistent with protein leakage from damaged glomeruli and increased protein overload in tubular lumens. This phenomenon promotes precipitation of TammHorsfall protein which is the major constituent of hyaline casts and the most abundant protein expressed in urine [10]. The function of Tamm-Horsfall protein relative to doxorubicin nephrotoxicity in the rat warrants further investigation. In the present study, other renal tubular findings were minimal; electron microscopy would be necessary to further characterize the glomerular lesions. 


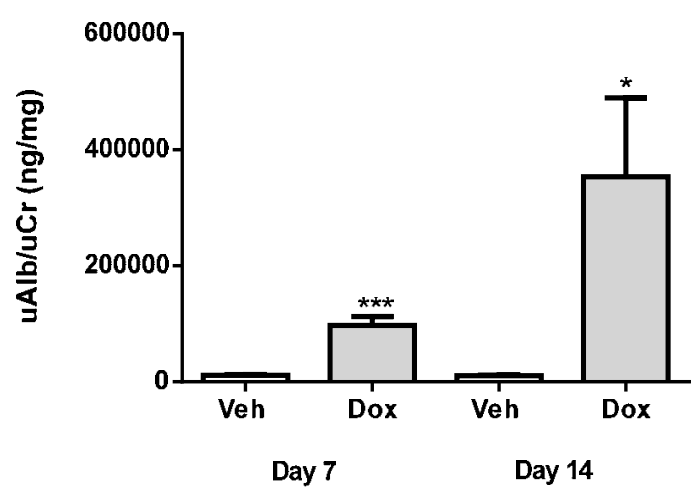

C

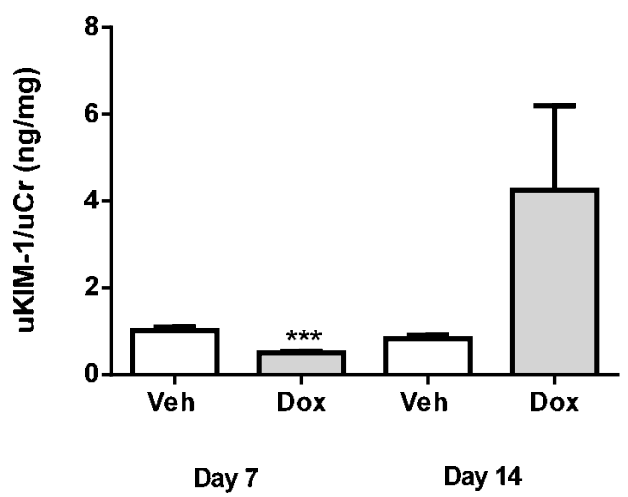

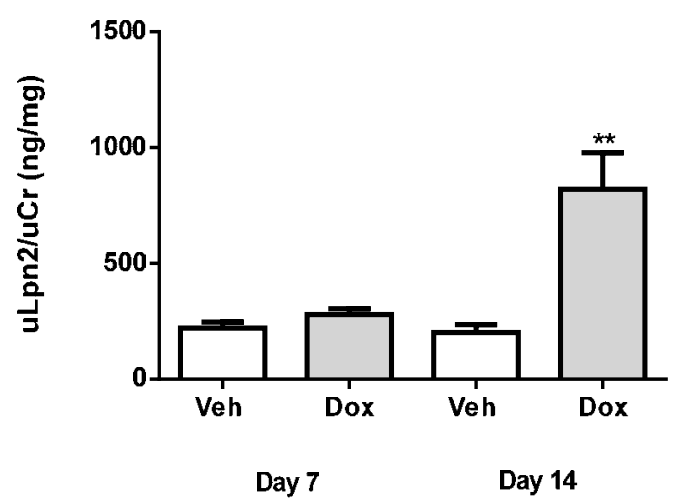

D

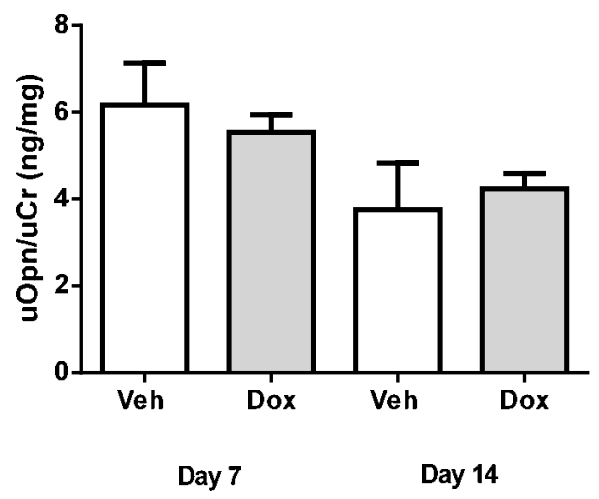

Figure 4: Changes in novel urinary biomarkers (urinary albumin, uAlb; urinary lipocalin-2, uLpn2; urinary kidney injury molecule 1, KIM-1; urinary osteopontin, uOpn) in male Sprague-Dawley rats administered Vehicle (Veh, $0.9 \%$ saline) or Doxorubicin (Dox, $5 \mathrm{mg} / \mathrm{kg} / \mathrm{dose}$ ) and samples collected on study days 7 and 14 . Urinary biomarkers were normalized to concurrent urinary creatinine $(\mathrm{uCr})$ concentrations. §Doxorubicin-induced increased uAlb concentrations were above the upper limit of assay detection. Values significantly different from vehicle control are indicated as ${ }^{* * *} p<0.001,{ }^{* *} p<0.01$ or ${ }^{*} p<0.05$.

In conclusion, multiplex measurement of urinary protein concentrations of uTP, uAlb, uKIM-1 and uOpn showed that increase adrenal biomarker concentration changes, particularly temporally increased $\mathrm{uAlb} / \mathrm{uCr}$ and decreased $\mathrm{uKIM}-1 / \mathrm{uCr}$ support opportunities to monitor for subclinical doxorubicin-induced early, acute glomerular injury with secondary renal tubular injury in male Sprague-Dawley rats. Furthermore, in this model, uMAlb/uCr and uAlb/uCr were more sensitive than $\mathrm{sCr}$ and $\mathrm{BUN}$ as well as $\mathrm{uTP} / \mathrm{uCr}, \mathrm{uOpn} / \mathrm{uCr}$ and $\mathrm{uLpn} 2 /$ $\mathrm{uCr}$ in the early prediction of progressive doxorubicin nephrotoxicity. While significant proteinuria which may be more specifically characterized by increased urinary albumin has long been associated with DIKI, urinary albumin had not been shown as a specific indicator of location of compound-related renal injury (glomerulus versus tubules). In this study, we have shown that increased $\mathrm{uMAlb} / \mathrm{uCr}$ and $\mathrm{uAlb} / \mathrm{uCr}$ in the absence of increased $\mathrm{uKIM}-1 / \mathrm{uCr}$ is predictive of a predominant compound-induced glomerular injury in rats. This phenomenon should be evaluated with other nephrotoxicants as well as in higher species. Additional studies may lead to the identification and validation of novel biomarkers that may be used as sensitive and specific genomic and/or proteomic biomarkers to monitor druginduced nephrotoxicity in preclinical studies in the rat and/or the clinical setting.

\section{Acknowledgements}

We would like to thank Antonio Guy and Matthew Nemec for conducting the in life study phase, Lynn Varacallo for conducting the clinical pathology parameter measurements, and Monica Singer for conducting the novel renal biomarker measurements and the Renal Integrated Safety Assessment team within Janssen Research \& Development, L.L.C. for their support.

\section{References}

1. Dieterle F, Sistare F, Goodsaid F, Papaluca M, Ozer JS, et al. (2010) Renal biomarker qualification submission: a dialog between the FDA EMEA and Predictive Safety Testing Consortium. Nat Biotechnol 28: 455-462.

2. Shu Y, Hoshi S, Tomari S, Watanabe T, Nagata M (2002) Phenotypic changes and cell cycle activation in early tubulointerstitial injury of rat adriamycin nephrosis. Pathol Int 52: 214-223.

3. Bertani T, Poggi A, Pozzoni R, Delaini F, Sacchi G, et al. (1982) Adriamycininduced nephrotic syndrome in rats: sequence of pathologic events. Lab Invest 46: 16-23.

4. Dodiya H, Jain M, Goswami S (2011) Study of urinary biomarkers for nephrotoxicityin Wistar rats. Journal of Pharmacology and Toxicology 6: 571579 .

5. Pippin JW, Brinkkoetter PT, Cormack-Aboud FC, Durvasula RV, Hauser PV et al. (2009) Inducible rodent models of acquired podocyte diseases. Am J Physiol Renal Physiol 296: F213-229.

6. Shu Y, Hoshi S, Tomari S, Watanabe T, Nagata M (2002) Phenotypic changes 
Citation: McDuffie JE, Sonee M, Ma J, Almy F, Liu X, et al. (2014) Feasibility of Protein Biomarkers in the Prediction of Subclinical Doxorubicin Nephrotoxicity in Male Sprague-Dawley Rat. J Mol Biomark Diagn 5: 165. doi:10.4172/2155-9929.1000165

and cell cycle activation in early tubulointerstitial injury of rat adriamycin nephrosis. Pathol Int 52: 214-223.

7. Xie HG, Wang SK, Cao CC, Harpur E (2013) Qualified kidney biomarkers and their potential significance in drug safety evaluation and prediction. Pharmaco Ther 137: 100-107.

8. Joles JA, Bijleveld C, van Tol A, Geelen MJ, Koomans HA (1995) Plasma triglyceride levels are higher in nephrotic than in analbuminemic rats despite a similar increase in hepatic triglyceride secretion. Kidney Int 47: 566-572.

9. McDuffie JE, Ma JY, Sablad M, Sonee M, Varacallo L, et al. (2013) Time course of renal proximal tubule injury, reversal, and related biomarker changes in rats following Cisplatin administration. Int J Toxicol 32: 251-260.
10. Xie Y, Sakatsume M, Nishi S, Narita I, Arakawa M, et al. (2001) Expression, roles, receptors, and regulation of osteopontin in the kidney. Kidney Int 60 : 1645-1657.

11. Langelueddecke C, Roussa E, Fenton RA, Wolff NA, Lee WK, et al. (2012) Lipocalin-2 (24p3/neutrophil gelatinase-associated lipocalin (NGAL)) receptor is expressed in distal nephron and mediates protein endocytosis. J Biol Chem 287: $159-169$

12. Bauchet AL, Masson R, Guffroy M, Slaoui M (2011) Immunohistochemica identification of kidney nephron segments in the dog, rat, mouse, and cynomolgus monkey. Toxicol Pathol 39: 1115-1128.

This article was originally published in a special issue, Biomarkers: Toxicology handled by Editor(s). Dr. James V. Rogers, Wright State University, USA; Dr. Jagiit S. Yadav, University of Cincinnati, USA; Dr. Huixiao Hong, National Center for Toxicological Research, USA 